\section{Diurnal variation in microcirculation of ocular fundus and visual field change in normal-tension glaucoma}

T Okuno, T Sugiyama, S Kojima, M Nakajima and T Ikeda

\section{Abstract}

Purpose Diurnal variations in microcirculation of the ocular fundus in normal-tension glaucoma (NTG) were examined to compare with the normal control eyes. The correlation between progression of visual field impairment and diurnal variations in ocular circulation was also studied.

Methods The subjects were 12 patients with NTG and 12 normal controls. Blood pressure (BP), intraocular pressure (IOP), ocular perfusion pressure (OPP), and square blur rate (SBR), an index of microcirculation acquired by the laser speckle method, were measured at 9 a.m. (morning), 3 p.m. (afternoon), and 9 p.m. (night). Diurnal variations in SBR were tested using the Friedman test and Wilcoxon signed ranks test. On the other hand, diurnal variations in BP, IOP, and OPP were tested by the analysis of variance. The visual field was evaluated at the same time as determining diurnal variation and again about 9 months later to calculate the change. The correlation between variation ratio in SBR and the change in visual field was examined by simple regression.

Results A significant decrease $(P=0.04)$ was found in SBR at night, as compared with the morning value, in the optic nerve head (ONH) of NTG, although no significant diurnal variations were found in SBR either in the choroid-retina or in normal control eyes. Other parameters showed no significant diurnal variations. The larger diurnal variation was in SBR of the $\mathrm{ONH}$, and the more exacerbated visual field impairment was $(r=0.59, P=0.04)$. Conclusion These findings suggest that diurnal variations in the microcirculation of the ONH may play a role in the progression of NTG.
Eye (2004) 18, 697-702. doi:10.1038/sj.eye.6700749

Published online 16 January 2004

Keywords: normal-tension glaucoma; diurnal variation; microcirculation; optic nerve head; choroid-retina; visual field

\section{Introduction}

In addition to mechanical damages due to high intraocular pressure (IOP), abnormal microcirculation in the optic nerve head $(\mathrm{ONH})$ has also been suggested as a cause of $\mathrm{ONH}$ impairment in glaucoma. ${ }^{1-5}$ Recent studies have shown that nocturnal hypotension is often seen in glaucoma, especially normal-tension glaucoma (NTG), and these studies hypothesized that impaired circulation in the $\mathrm{ONH}$ plays a role in the pathogenesis of NTG. ${ }^{6-10}$ In this study, we compared diurnal variations in the ocular microcirculation of NTG and normal eyes, and studied the relation between these diurnal variations and the progression of visual field impairment in NTG.

\section{Materials and methods}

\section{Subjects}

All subjects were outpatients recruited from the Department of Ophthalmology at Osaka Medical College. The institutional review board of Osaka Medical College approved consent forms for this study. All subjects were briefed on the procedure and signed informed consent forms before the measurements were taken. The subjects were enrolled in 1998 and 1999.

The NTG patients in this study met the following criteria: (1) IOP was always recorded as $15 \mathrm{mmHg}$ or less; (2) normal open angle; (3) glaucomatous cupping in the $\mathrm{ONH}$ (cup/ONH ratio of 0.6 or more); (4) glaucomatous visual
Department of Ophthalmology Osaka Medical College Osaka, Japan

Correspondence:

T Sugiyama

Department of

Ophthalmology

Osaka Medical College

2-7 Daigaku-cho

Takatsuki

Osaka 569-8686

Japan

Tel: +81726831221

Fax: +81726818195

E-mail: opt017@

poh.osaka-med.ac.jp

Received: 15 January 2003 Accepted: 26 June 2003 Published online: 16 January 2004

This work was previously presented at American Academy of Ophthalmology held in Daras on 22-25 of October, 2000. 
field loss detected by a Humphrey Field Analyzer; (5) no lesion potentially causing optic nerve disturbance detected in the brain or paranasal sinus by CT scans; and (6) no history of serious hypotension or bleeding.

The subjects were 12 NTG patients (five males, seven females) and 12 normal controls (three males, nine females) who had no ocular abnormality other than weak refractive errors or mild cataracts. Patients with diabetes mellitus or systemic cardiovascular diseases, smokers, and patients taking antihypertensive agents, antiglaucoma drugs, or other medications that might affect ocular circulation were excluded from both groups. The subjects in whom measurement was impeded by opacity due to cataracts or other optic media were excluded from both groups, as well. The NTG patients whose reproducibility was poor in the previous $2.8 \pm 0.9$ measurements (for each patient) of visual fields were also excluded. The ages in both groups were matched (NTG group: $64.9 \pm 10.0$ years, normal control group: $64.3 \pm 9.4$ years as mean $\pm S D$ at determination of diurnal variations). There was no significant difference in IOP between the two groups (NTG group: $13.1 \pm 1.6 \mathrm{mmHg}$, normal control group: $14.3 \pm 2.8 \mathrm{mmHg}$ as mean $\pm \mathrm{SD}$ at determination of diurnal variations). The stage of progression in the NTG group ranged from early to middle stages as judged by a Humphrey Field Analyzer Central 30-2 full threshold program (Humphrey Instruments, San Leandro, CA, USA). The mean deviation (MD) was $-4.75 \pm 5.42 \mathrm{~dB}$ as mean $\pm \mathrm{SD}$ at the time of measurement of ocular blood flow. In the NTG group, the eye with the lower MD value was chosen for measurement of diurnal variations of blood flow. In the normal control group, eyes with clear lenses were randomly chosen for the measurement.

\section{SBR measurements in ONH and choroid-retina}

The mechanism of the laser speckle tissue circulation analyser has been described in other articles. ${ }^{11-16}$ The system consists of a fundus camera (TRC-WT3, Topcon, Tokyo, Japan) equipped with a diode laser (wavelength $808 \mathrm{~nm}$ ). The scattered laser light is imaged on a sensor $(100 \times 100$ pixels, BASIS type, Canon, Tokyo, Japan), which corresponds to the $0.72 \times 0.72 \mathrm{~mm}$ field $\left(30^{\circ}\right.$ visual angle) in the human ocular fundus where the speckle pattern appears. The mean difference between the mean pulse intensity over 98 scans (I mean) and each pixel pulse intensity is calculated; the ratio of I mean to this mean difference is defined as normalized blur (NB). The NB is the approximate reciprocal of speckle contrast and is regarded as a quantitative index of tissue blood velocity. Its relative change correlates well with that in the tissue blood flow measured by a hydrogen clearance method in the ONH. ${ }^{13}$ The square blur rate (SBR) value is another index obtained from $(\mathrm{NB})^{2}$ and is considered to correlate to blood flow better than NB. ${ }^{16}$

After the administration of one drop of $0.4 \%$ tropicamide (Mydrin $\mathrm{M}^{\mathbb{R}}$, Santen Pharmaceutical, Osaka, Japan) for mydriasis, a photograph of ocular fundus was taken in order to locate and record each measurement area. The SBR values in $\mathrm{ONH}$ and choroid-retina, blood pressure (BP) and IOP were measured at 9 a.m. (morning), 3 p.m. (afternoon), and 9 p.m. (night). Each subject fasted for at least $2 \mathrm{~h}$ prior to measurement. The image speckles from the field located in the temporal $\mathrm{ONH}$, and those from the field between the macula and the $\mathrm{ONH}$, free from surface vessels, were recorded for measurement of the SBR values in the $\mathrm{ONH}$ and in the choroid-retina, respectively. The SBR values in the $\mathrm{ONH}$ and choroid-retina were calculated every $0.125 \mathrm{~s}$ for $7 \mathrm{~s}$ and averaged for 4-6 cardiac cycles to obtain their mean SBR values. Among morning, afternoon, and night SBR, the maximum $\left(\mathrm{SBR}_{\max }\right)$ and minimum $\left(\mathrm{SBR}_{\min }\right)$ were determined, and the variation ratio in SBR was calculated using the following equation:

Variation ratio in $\mathrm{SBR}=\mathrm{SBR} \max / \mathrm{SBR} \min$

\section{Measurement of diurnal variations in BP and IOP}

Brachial artery BP and pulse rate were measured using an automated sphygmomanometer (JENTOW-7700 (CS), Nippon Colin Co., Ltd, Tokyo, Japan) after each determination of SBR. The mean blood pressure $\left(\mathrm{BP}_{\mathrm{m}}\right)$ was calculated from systolic $\mathrm{BP}\left(\mathrm{BP}_{\mathrm{s}}\right)$ and diastolic $\mathrm{BP}$ $\left(\mathrm{BP}_{\mathrm{d}}\right)$, according to the following equation.

$$
\mathrm{BP}_{\mathrm{m}}=\mathrm{BP}_{\mathrm{d}}+1 / 3\left(\mathrm{BP}_{\mathrm{s}}-\mathrm{BP}_{\mathrm{d}}\right)
$$

IOP was also measured with a Goldmann applanation tonometer after each determination of SBR. Using the above values of IOP and $\mathrm{BP}_{\mathrm{m}}$, the ocular perfusion pressure (OPP) was calculated using the following equation.

$$
\mathrm{OPP}=2 / 3 \mathrm{BP}_{\mathrm{m}}-\mathrm{IOP}
$$

\section{Measurement of change in visual field}

For the NTG group, the visual field was measured near the determination of diurnal variations and 5-14 $(8.8 \pm 3.1$ as mean $\pm S D)$ months later, using a Humphrey Field Analyzer (Program: Central 30-2). The change in visual field, $\triangle \mathrm{MD}$ (mean deviation), was calculated from these values of MD (pre) and MD (post), using the equation below.

$$
\Delta \mathrm{MD}=\mathrm{MD}(\text { post })-\mathrm{MD}(\text { pre })
$$

During this period, the patient was not treated with antiglaucoma drugs or calcium antagonists. 


\section{Statistical analysis}

Since SBR, an index of relative blood velocity, does not necessarily follow a normal distribution, it was treated as a nonparametric variable. The diurnal variations in SBR were tested for significance using the Friedman test. If a significant difference was detected, the differences between morning, afternoon, and night values were analysed by the Wilcoxon signed ranks test.

Since the data for BP, IOP, and OPP would show approximately normal distributions, the diurnal variations in these variables were tested by the analysis of variance (ANOVA).

The correlation coefficients were determined for variation ratio in SBR and $\triangle \mathrm{MD}$ for the $\mathrm{ONH}$ and choroid-retina by simple regression. The correlation between the interval of visual field measurements and $\Delta \mathrm{MD}$ was also examined by simple regression.

In all cases, $P<0.05$ was regarded as indicating a statistical significance, and $P<0.1$ as indicating a tendency.

\section{Results}

Diurnal variations in the microcirculation in the ocular fundus, IOP, and BP

Diurnal variations of SBR in the $\mathrm{ONH}$ are shown in Figure 1. In the NTG group, there was a significant difference in diurnal SBR (Friedman test, $P=0.02$ ).

Furthermore, SBR was significantly lower in the night, as compared with the morning (Wilcoxon signed ranks test, $P=0.04)$. There was also a tendency for SBR to be lower in the afternoon than in the morning $(P=0.06)$. In the normal control group on the other hand, no significant differences in diurnal variation were seen (Friedman test, $P>0.1)$.

Diurnal variations of SBR in the choroid-retina are shown in Figure 2. In both the NTG and normal control groups, there were no significant differences in diurnal variations of SBR (Friedman test, $P>0.1$ ), although in the NTG group, SBR seemed lower in the afternoon and night.

Diurnal variations in IOP, BP, and OPP are shown in Table 1. Statistical analysis by ANOVA yielded no significant differences between the NTG group and normal control group $(P>0.1)$. In the NTG group, however, afternoon and night OPP were slightly lower than morning OPP.

\section{Diurnal variations in SBR and visual field changes}

Scatter plots of the variation ratio in SBR and $\triangle \mathrm{MD}$ are presented in Figures 3 and 4. The correlation coefficient for the ONH was $r=0.59(P=0.04)$ and for the

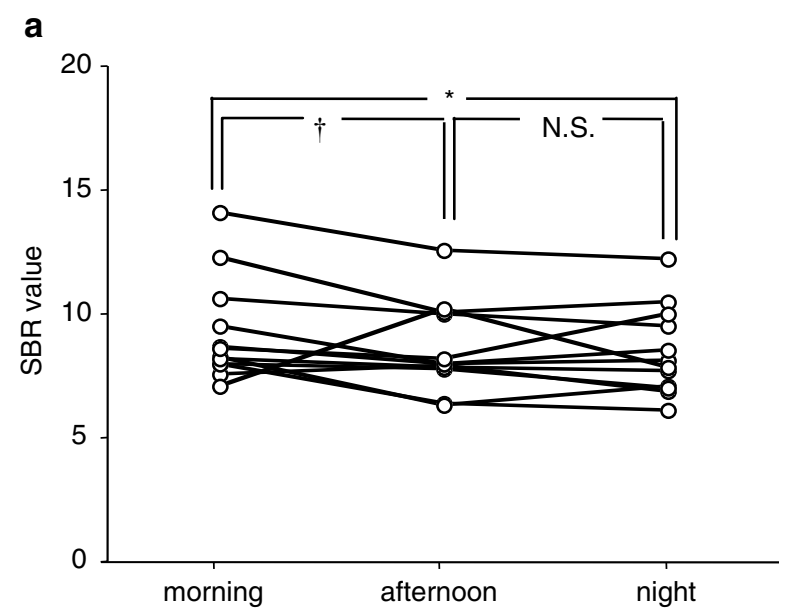

b

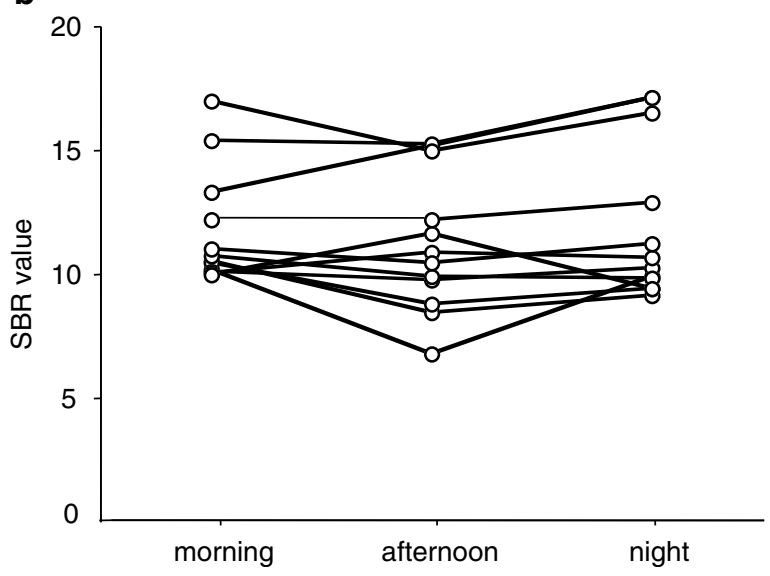

Figure 1 Diurnal variations in SBR in the ONH: (a) NTG group, (b) normal control group. Morning, afternoon, and night SBR are significantly different in the NTG group (Friedman test, $P=0.02$ ). Compared with morning SBR, night SBR is significantly lower (Wilcoxon signed ranks test, ${ }^{*} P=0.04$ ) and afternoon SBR has a tendency to be lower $\left({ }^{\dagger} P=0.06\right)$. There is no significant difference between afternoon and night SBR. In the normal control group by contrast, there are no significant differences between morning, afternoon, or night SBR.

choroid-retina, $r=0.23(P=0.47)$. Hence, the correlation was significant for the $\mathrm{ONH}$. The correlation coefficient between the interval of visual field measurements and $\Delta \mathrm{MD}$ was not significant $(r=0.35, P=0.27)$.

\section{Discussion}

There have been recent reports that nocturnal hypotension is frequently manifested in NTG, as well as in glaucoma with well-controlled IOP. ${ }^{6-10}$ Other reports have found that nocturnal hypotension was frequently found in glaucoma with progressive visual field impairment. ${ }^{17}$ In contrast, there is a report that nocturnal decrease in both BP and blood velocity in the ophthalmic artery was not detected in primary open-angle glaucoma 


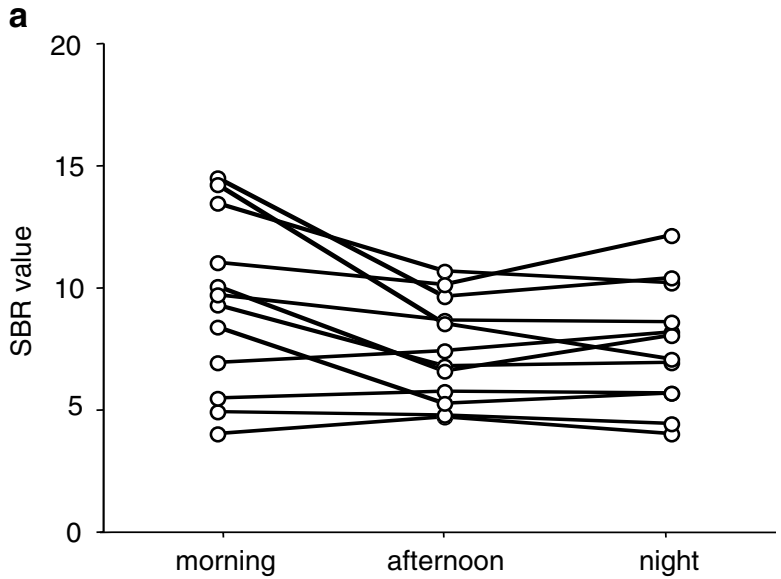

b

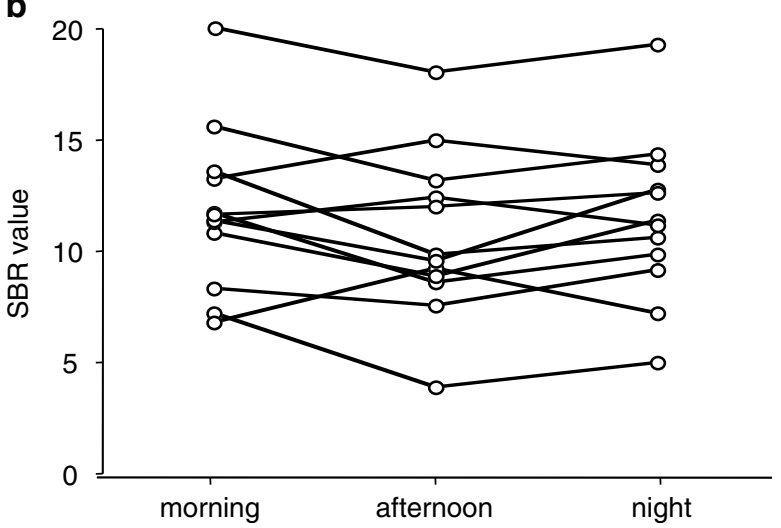

Figure 2 Diurnal variations in SBR in the choroid-retina: (a) NTG group, (b) normal control group. There are no significant differences between morning, afternoon, or night SBR in either the NTG group or normal control group (Friedman test, $P>0.05)$.

Table 1 Diurnal variations of hemodynamic parameters

\begin{tabular}{lccc}
\hline & Morning & Afternoon & Night \\
\hline (a) Normal control & & & \\
BPm (mmHg) & $99 \pm 5$ & $94 \pm 5$ & $94 \pm 4$ \\
OP (mmHg) & $14.8 \pm 1.0$ & $14.1 \pm 0.8$ & $13.9 \pm 0.9$ \\
OPP (mmHg) & $50 \pm 3$ & $50 \pm 3$ & $50 \pm 3$ \\
\hline (b) NTG & & & \\
BPm (mmHg) & $97 \pm 5$ & $96 \pm 4$ & $96 \pm 4$ \\
IOP (mmH) & $14.1 \pm 0.7$ & $13.0 \pm 0.6$ & $12.3 \pm 0.5$ \\
OPP (mmHg) & $52 \pm 3$ & $50 \pm 3$ & $50 \pm 2$ \\
\hline
\end{tabular}

BPm: mean blood pressure, IOP: intraocular pressure, OPP: ocular perfusion pressure, mean \pm SE $(n=12)$. There are no significant differences between morning, afternoon, or night values in either the NTC group or normal control group $(P>0.1$, Friedman test).

with stable visual field and without systemic hypertension. ${ }^{18}$ One possible cause of ONH impairment in glaucoma is a microcirculation disorder in the ocular fundus. ${ }^{1-5}$ On the other hand, it is possible that diurnal variations in $\mathrm{BP}$ cause those variations in

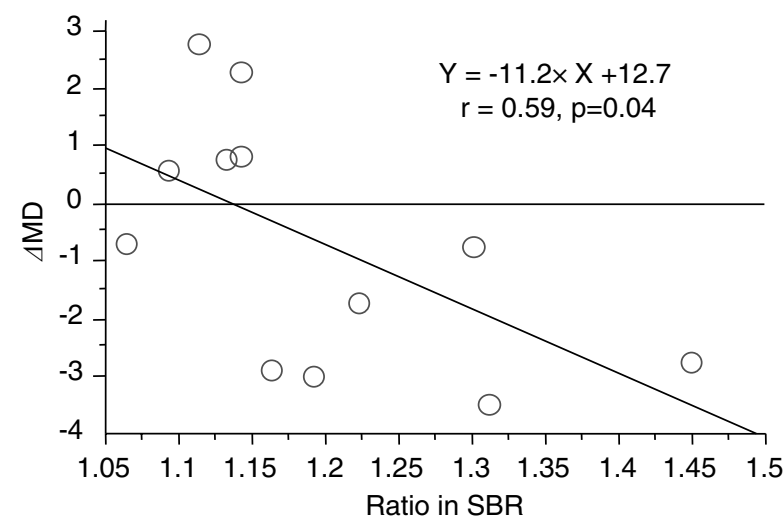

Figure 3 Diurnal variation ratio in SBR in the $\mathrm{ONH}$ and changes in the visual field (NTG group only). There is a significant and evident correlation $(r=0.59, P=0.04)$.

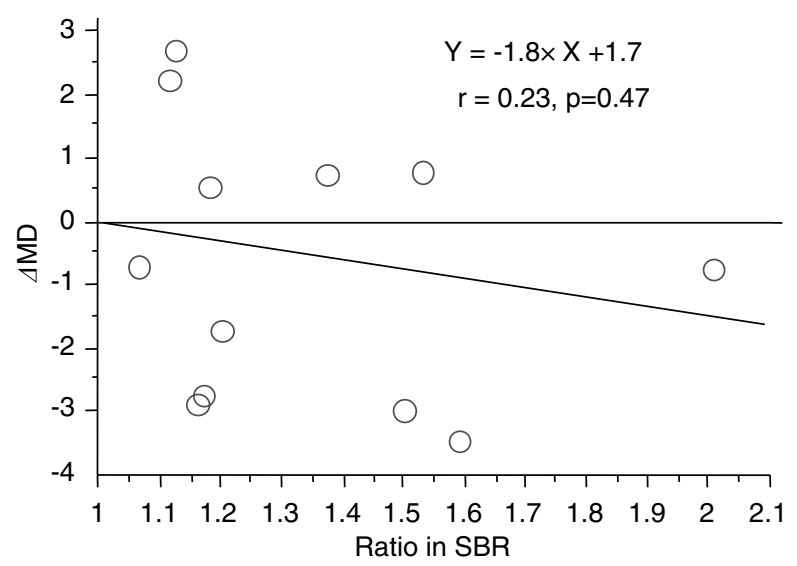

Figure 4 Diurnal variation ratio in the choroid-retina and changes in the visual field (NTG group only). There is a weak correlation but not significant $(r=0.23, P=0.47)$.

microcirculation of the ocular fundus. However, no reports accounted for the involvement of diurnal variations in the ocular microcirculation in the progression of NTG. Therefore, in this study, we set out to measure these diurnal variations in the microcirculation of the ocular fundus.

In the current study, we used a recently developed laser speckle circulation analyser to evaluate the microcirculation in the ocular fundus. In animal experiments, it has been shown that the changes in tissue blood flow obtained by a hydrogen clearance method or a microsphere method and the changes in NB obtained by the laser speckle method are significantly correlated for the $\mathrm{ONH}$ and choroid-retina. ${ }^{13,14}$ In human eyes, adequate reproducibility has been demonstrated in the measurement of $\mathrm{ONH}$ microcirculation. ${ }^{15}$ As SBR values in the $\mathrm{ONH}$ vary with age, ${ }^{19}$ we designed the present investigation in groups of approximately age-matched 
subjects. We note, however, that SBR values vary considerably in different eyes; this may be due to a difference in the reflection or transmission of the laser beam in different eyes. ${ }^{13}$ Therefore, we compared the changes in SBR values of the same eyes in the current study.

We found that $\mathrm{ONH}$ microcirculation was significantly lower at night, as compared with in the morning, in NTG eyes, although we did not find any significant diurnal variations in microcirculation of control ocular fundus. OPP was slightly lower at night than in the morning in NTG eyes, but not to a significant degree. Given these findings, it is suggested that the mechanism regulating ocular microcirculation may be dysfunctional in NTG eyes.

In the choroid-retina, however, no significant diurnal variations were seen in either the NTG or normal control groups. Investigations of monkeys (a model of retinal artery occlusion) and of humans (retinal artery occlusion, geographic choroiditis) have shown that at least $75 \%$ of the NB obtained from the choroid-retina is indicative of choroidal blood flow. ${ }^{12}$ Hence, the NB and SBR values of the choroid-retina may be readily affected by OPP. No significant diurnal variations were produced in the choroid-retinal microcirculation probably because of no significant diurnal changes in OPP in either the NTG or normal control groups in the present study.

BP and OPP both fell slightly in the afternoon and night in the NTG group, however, failed to reveal any significant diurnal variations in either the NTG group or normal control group. This differed from the findings in numerous published reports, ${ }^{6-9,17}$ in which a decreased $\mathrm{BP}$ was noted in the night. In our study, the measurement time in the night was 9 p.m., not as late as in the other researches, which might be the cause of failure to observe a significant decrease in BP or OPP.

It could be speculated that the regulatory function for ocular circulation was more impaired in NTG patients with larger diurnal variations in ocular microcirculation. We therefore investigated the relationship between variation ratio in SBR and a change in visual field. A significant correlation $(r=0.59, P=0.04)$ was found between the variation ratio in SBR for the $\mathrm{ONH}$ and $\triangle \mathrm{MD}$, although no correlation was found between the interval of visual field measurements and $\triangle \mathrm{MD}$.

However, there was no significant correlation between the variation ratio in SBR for the choroid-retina and $\triangle \mathrm{MD}$ ( $r=0.23, P=0.47)$. It can be predicted that progression of visual field impairment will be more rapid in eyes showing greater diurnal variations in microcirculation of the ONH. Hence, it might be concluded that the patient in whom autoregulation of $\mathrm{ONH}$ circulation is impaired might be more readily predisposed to progress in visual field impairment.

\section{Acknowledgements}

We thank Yasuichiro Nishimura PhD, Department of Mathematics, Osaka Medical College, for his helpful suggestions regarding the statistical analysis.

\section{References}

1 Anderson DR. Introductory comments on blood flow autoregulation in the optic nerve head and vascular risk factors in glaucoma. Surv Ophthalmol 1999; 43 (Suppl 1): S5-S9.

2 Chung HS, Harris A, Evans DW, Kagemann L, Garzozi HJ, Martin B. Vascular aspects in the pathophysiology of glaucomatous optic neuropathy. Surv Ophthalmol 1999; 43 (Suppl 1): S43-S50.

3 Flammer J, Orgül S. Optic nerve blood-flow abnormalities in glaucoma. Prog Retin Eye Res 1998; 17: 267-289.

4 Grunwald JE, Piltz J, Hariprasad SM, DuPont J. Optic nerve and choroidal circulation in glaucoma. Invest Ophthalmol Vis Sci 1998; 39: 2329-2336.

5 Michelson G, Langhans MJ, Harazny J, Dichtl A. Visual field defect and perfusion of the juxtapapillary retina and the neuroretinal rim area in primary open-angle glaucoma. Graefes Arch Clin Exp Ophthalmol 1998; 236: 80-85.

6 Claridge KG, Smith SE. Diurnal variation in pulsatile ocular blood flow in normal and glaucomatous eyes. Surv Ophthalmol 1994; 38 (Suppl): S198-S205.

7 Hayreh SS, Zimmerman MB, Podhajsky P, Alward WL. Nocturnal arterial hypotension and its role in optic nerve head and ocular ischemic disorders. Am J Ophthalmol 1994; 117: 603-624

8 Kaiser HJ, Flammer J. Systemic hypotension: a risk factor for glaucomatous damage? Ophthalmolgica 1991; 203: 105-108.

9 Meyer JH, Brandi-Dohrn J, Funk J. Twenty four hour blood pressure monitoring in normal tension glaucoma. $\mathrm{Br} J$ Ophthalmol 1996; 80: 864-867.

10 Orgül S, Kaiser HJ, Flammer J, Gasser P. Systemic blood pressure and capillary blood-cell velocity in glaucoma patients: a preliminary study. Eur J Ophthalmol 1995; 5: 88-91.

11 Fujii H. Visualization of retinal blood flow by laser speckle flowgraphy. Med Biol Eng Comput 1994; 32: 302-304.

12 Isono H, Kimura Y, Aoyagi K, Fujii H, Konishi N. Analysis of choroidal blood flow by laser speckle flowgraphy. Nippon Gannka Gakkai Zasshi (J Jpn Ophthalmol Soc) 1997; 101: 684-691.

13 Sugiyama T, Utsumi T, Azuma I, Fujii H. Measurement of optic nerve head circulation: comparison of laser speckle and hydrogen clearance methods. Jpn J Ophthalmol 1996; 40: 339-343.

14 Tamaki Y, Araie M, Kawamoto E, Eguchi S, Fujii H. Noncontact, two-dimensional measurement of tissue circulation in choroid and optic nerve head using laser speckle phenomenon. Exp Eye Res 1995; 60: 373-384.

15 Tamaki Y, Araie M, Tomita K, Nagahara M, Tomidokoro A, Fujii H. Real-time measurement of human optic nerve head and choroid circulation using the laser speckle phenomenon. Jpn J Ophthalmol 1997; 41: 49-54.

16 Fujii H, Konishi N, Okamoto K, Dobashi Y. A new version of real-time laser flowgraphy. Atarashii Ganka (Eye) 1996; 13: 957-961. 
17 Graham SL, Drance SM, Wijsman K, Douglas GR, Mikelberg FS. Ambulatory blood pressure monitoring in glaucoma. Ophthalmology 1995; 102: 61-69.

18 Harris A, Spaeth G, Wilson R, Moster M, Sergott R, Martin B. Nocturnal ophthalmic arterial hemodynamics in primary open-angle glaucoma. J Glaucoma 1997; 6: 170-174.
19 Nagaya K, Takahashi H, Akiya S, Fujii H. Age-related changes in normal optic nerve head $(\mathrm{ONH})$ microcirculation assessed using laser speckle phenomenon. Atarashii Ganka (Eye) 1998; 15: 1465-1469. 